\title{
Impact of the Langdon effect on crossed-beam energy transfer
}

\author{
David Turnbull $^{1 *}$, Arnaud Colaïtis ${ }^{2}$, Aaron M. Hansen ${ }^{1}$, Avram L. Milder ${ }^{1}$, John P. Palastro ${ }^{1}$, \\ Joseph Katz ${ }^{1}$, Christophe Dorrer ${ }^{1}$, Brian E. Kruschwitz ${ }^{1}$, David J. Strozzi ${ }^{3}$, and Dustin H. Froula ${ }^{1}$
}

October 15, 2019

\author{
${ }^{1}$ University of Rochester Laboratory for Laser Energetics, Rochester, New York 14623, USA \\ ${ }^{2}$ Centre National da la Recherche Scientifique, Talence, France \\ ${ }^{3}$ Lawrence Livermore National Laboratory, Livermore, California 94550, USA
}

The prediction that laser plasma heating distorts the electron distribution function away from Maxwellian and towards a super-Gaussian distribution dates back four decades [1]. In conditions relevant to inertial confinement fusion, however, no direct evidence of this so-called "Langdon effect" has previously been observed. Here, measurements of the spatially and temporally resolved Thomson scattering spectrum indicate the presence of super-Gaussian electron distribution functions that are consistent with existing theory [2]. In such plasmas, ion acoustic wave frequencies increase monotonically with the super-Gaussian exponent [3]. Our results show that the measured power transfer between crossed laser beams mediated by ion acoustic waves requires a model that accounts for the non-Maxwellian electron distribution function, whereas the standard Maxwellian calculations overpredict power transfer over a wide region of parameter space. Including this effect is expected to improve the predictive capability of crossed-beam energy transfer modeling at the National Ignition Facility and may restore a larger operable design space for inertial confinement fusion experiments. This is also expected to motivate further inquiry in other areas impacted by non-Maxwellian electron distribution functions, such as laser 


\section{absorption, heat transport, and x-ray spectroscopy.}

Laser fusion experiments require many overlapping laser beams to propagate through long, underdense plasmas in order to precisely deposit their energy at desired locations, but laser-plasma interactions can complicate the intended result [4]. Crossed-beam energy transfer (CBET) is one example, whereby a frequency difference between two lasers in the plasma rest frame resonantly drives an ion acoustic wave (IAW) that scatters light from one beam to the other. With 96 beams overlapped in each laser entrance hole of an indirect-drive hohlraum, there are more than four thousand pairwise interactions that can induce a macroscopic redistribution of laser intensity within the hohlraum interior. The ability to manipulate this process via laser frequency detuning was initially seen as beneficial for designs with high initial hohlraum gas fill density, providing control over implosion symmetry while operating the National Ignition Facility (NIF) at its maximum energy [5-7]. However, when integrated observables indicated that there was less CBET than calculated, a tunable saturation clamp on IAW amplitudes was added to models [8], although the level $\left(\delta n_{e} / n_{e}=10^{-4}\right.$ to $\left.10^{-3}\right)$ was too small to be explained by known saturation mechanisms. Moreover, it varied between platforms - undermining the predictive capability of simulations. When the most comprehensive postmortem of high-gas-fill hohlraums concluded that time-dependent radiation flux asymmetry was a primary degradation mechanism - alone responsible for an $8 \times$ to $20 \times$ reduction in performance-errors in CBET calculations were implicated as one possible cause [9]. Several attempts to improve CBET modeling by including feedback between laser-plasma instabilities and hydrodynamics have reduced, but not eliminated, the discrepancies between experiments and unclamped simulations $[8,10]$.

The indirect-drive inertial confinement fusion (ICF) program has since shifted its emphasis to hohlraums with lower initial gas fill density, in large part, to minimize CBET and backscatter instabilities [11-13]. However, the freer expansion of ablated material from the high-Z hohlraum wall necessitates the use of shorter-duration laser pulsesconstricting the available design space — and predictive implosion modeling remains challenging. While symmetry is now primarily controlled without resorting to frequency detuning, calculations indicate that CBET can still significantly impact symmetry due to flow-induced Doppler shifts inside the hohlraum. Furthermore, some of the low gas fill designs have in fact reintroduced small amounts of frequency detuning to help achieve a symmetric implosion [14]. 
Accurate CBET modeling therefore remains a top priority for achieving high neutron yields in indirect-drive ICF.

This motivated the development of a CBET platform at the University of Rochester's Laboratory for Laser Energetics, where a wavelength tunable laser (TOP9) was built to study CBET in a well-characterized quasi-stationary plasma [15-17]. Initial experiments reported here suggest that the Langdon effect may be responsible for overpredicting power transfer in indirect-drive ICF relevant conditions. The term comes from a 1980 Letter in which A. B. Langdon explained that inverse Bremsstrahlung absorption of electromagnetic radiation in plasma preferentially heats low energy electrons, distorting the electron distribution function (EDF) away from Maxwellian and toward a super-Gaussian of order $m=5$ [Ref. [1]]. He defined the scaling parameter,

$$
\alpha=Z_{e f f} v_{o s c}^{2} / v_{t h}^{2}
$$

where $Z_{\text {eff }}=\left\langle Z^{2}\right\rangle /\langle Z\rangle$ is the effective ion charge; $v_{\text {osc }}=0.256 \sqrt{I} \lambda[\mathrm{m} / \mathrm{s}]$ is the velocity of electrons oscillating in the laser field, with laser intensity $I$ in units of $\mathrm{W} / \mathrm{cm}^{2}$ and laser wavelength $\lambda$ specified in $\mu \mathrm{m}$; and $v_{t h}=\sqrt{T_{e} e / M_{e}}$ is the electron thermal velocity, where electron temperature $T_{e}$ is specified in $\mathrm{eV}, e$ is the elementary charge, and $M_{e}$ is the mass of an electron in $\mathrm{kg}$. The parameter $\alpha$ represents the ratio of inverse bremsstrahlung heating to electronelectron collisions, which act to restore a Maxwellian distribution. Subsequent Fokker-Planck simulations by J. P. Matte et al. [2] under a wide range of laser heating conditions confirmed that super-Gaussian EDF's are produced in the form

$$
f_{m}(v)=C_{m} \exp \left[-\left(v / v_{m}\right)^{m}\right]
$$

where $v_{m}^{2}=\frac{3 T_{e} e}{M_{e}} \frac{\Gamma(3 / m)}{\Gamma(5 / m)}, C_{m}=\frac{N_{e}}{4 \pi} \frac{m}{\Gamma(3 / m) v_{m}^{3}}, \Gamma$ is the gamma function, and

$$
m(\alpha)=2+3 /\left(1+1.66 / \alpha^{0.724}\right)
$$

is only a function of $\alpha$. Since EDF's in that form were first discussed by C. T. Dum in the context of ion sound turbulence $[18,19]$, they are sometimes referred to as Dum-Langdon-Matte, or DLM, distributions. Note that $\left\langle v^{2}\right\rangle=$ $3 T_{e} e / M_{e}$ for any $m$, meaning there is still a notion of temperature for these distribution functions. Equation 3 satisfied the known limits: $m \rightarrow 2$ for $\alpha \ll 1$ and $m \rightarrow 5$ for $\alpha \gg 1$. A microwave experiment claimed to validate this theory, albeit with density and temperature many orders of magnitude lower than those typical of ICF environments [20]. 


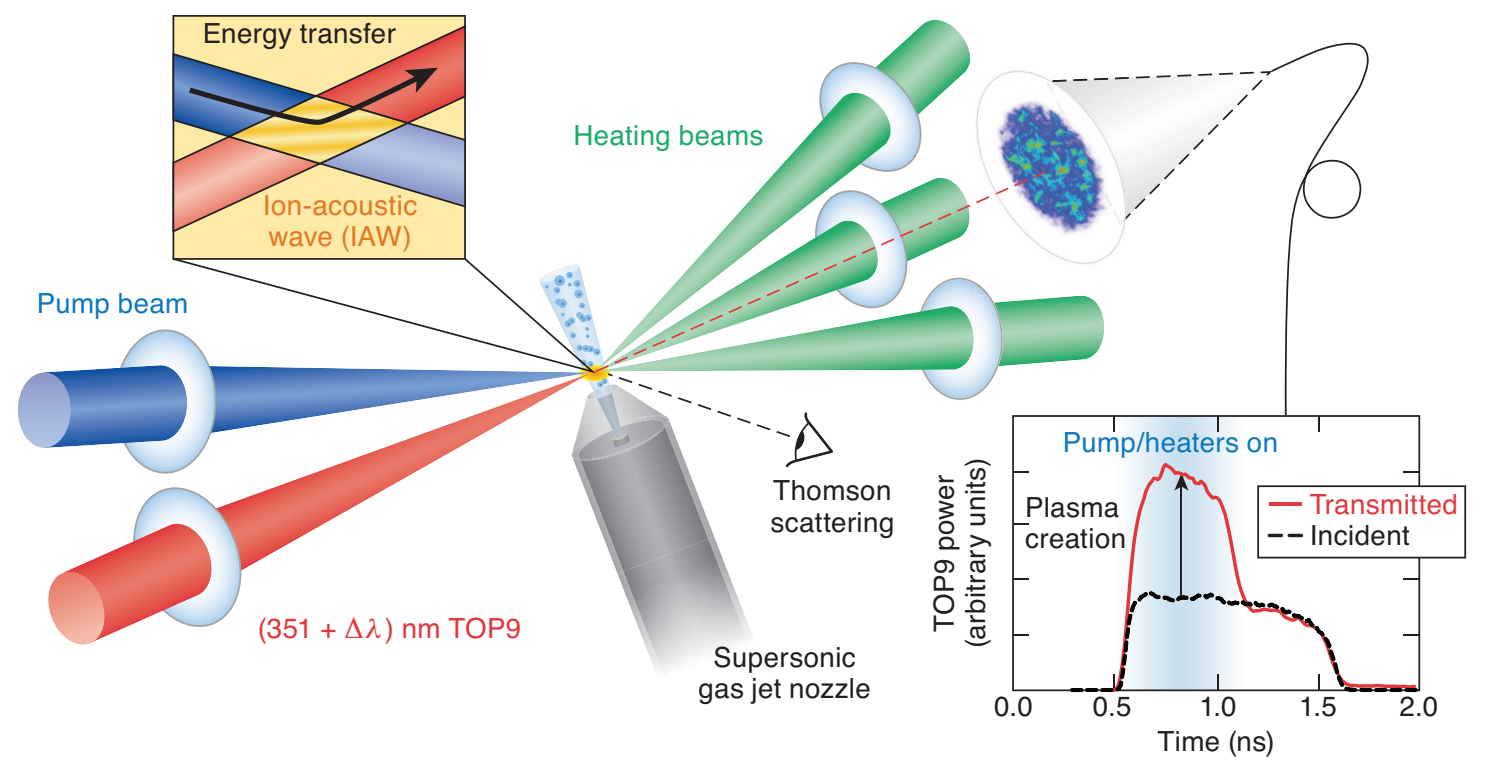

E28290J1

Figure 1: Platform for TOP9 experiments. A supersonic nozzle emitted a nitrogen/hydrogen gas mixture $1.3 \mathrm{~mm}$ from target chamber center. Nine beams, each $850 \mu \mathrm{m}$ in diameter, ionized and heated the gas in a quasi-symmetric manner for the first 500 ps. Over the next 500 ps, a single pump and TOP9 were turned on, and an ion acoustic wave mediated energy transfer between the two beams. Additional heater beams that nearly counter-propagated with TOP9 could be turned on during this time to accentuate the Langdon effect; such beams otherwise interacted minimally with the pump and TOP9 as a result of their wavelength and geometry. For the last 500 ps, TOP9 was the sole remaining beam, providing a baseline plasma transmission measurement. TOP9 terminated on a thin diffuser sheet that was measured in transmission by a CCD camera and fiber-coupled streaked spectrometer. 
Although it is often assumed that the Langdon effect only impacts absorption in high- $Z$ plasmas, such nonMaxwellian distribution functions are known to affect the dispersion relation that relates the frequency $\omega$ and wave number $k$ of ion acoustic waves [3],

$$
\omega=k c_{s}\left[\frac{3 \Gamma^{2}(3 / m)}{\Gamma(1 / m) \Gamma(5 / m)}\right]^{1 / 2},
$$

, where $c_{s}$ is the sound speed, which would directly impact CBET by shifting the ion acoustic resonance. The square root term modifies the usual dispersion relation and leads to a monotonic increase of IAW frequency with superGaussian order, which results from the smaller number of low energy electrons $[f(v \approx 0)]$ available to shield the ion oscillations. (Note that the sensitivity to low energy electrons is very analogous to the impact on laser absorption first highlighted by Langdon [1].)

Experiments were conducted as shown in Fig. 1. TOP9 was crossed with a single nearly co-propagating pump beam in a plasma that was preformed from a mixture of hydrogen and nitrogen gas. Its wavelength was red-shifted so as to extract energy from the pump, and its power was then diagnosed using a transmitted beam diagnostic. Results will be shown with and without nearly counter-propagating heater beams, which (when present) enhanced the Langdon effect without contributing significantly to the CBET gain.

Both spatially and temporally resolved Thomson scattering were used to characterize the plasma conditions in order to constrain the CBET modeling. To allow for a non-Maxwellian EDF, the electron susceptibility in the collisionless Thomson scattering spectral density function was computed numerically for a given super-Gaussian exponent.

We began by testing the intensity scaling of the Langdon effect. To do so, the number of beams present during the 0.7 to $1.2 \mathrm{~ns}$ period was varied from 1 to 4 . To simplify the analysis, only heaters were used because they all had nominally identical power and spatial smoothing (the much weaker TOP9 was also present, but impacted plasma conditions and scattering signals negligibly). Figure 2(a) shows the raw data for the three heater beam case. Both the electron plasma wave (EPW) and the ion acoustic wave features were the sum of the scattered light from all beams. (Note that scattering from the more numerous plasma-forming beams is visible for the first $500 \mathrm{ps}$.) The heaters had similar scattering angles with respect to the Thomson scattering collection system $\left(101.7^{\circ}, 102.8^{\circ}\right.$, and $\left.141.1^{\circ}\right)$ and 

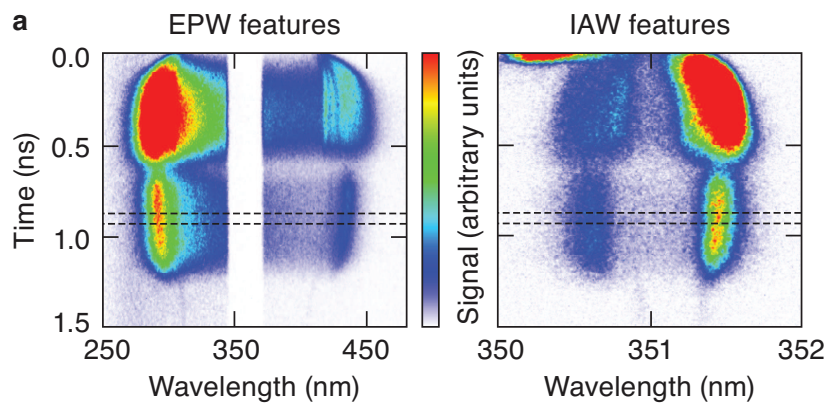

b

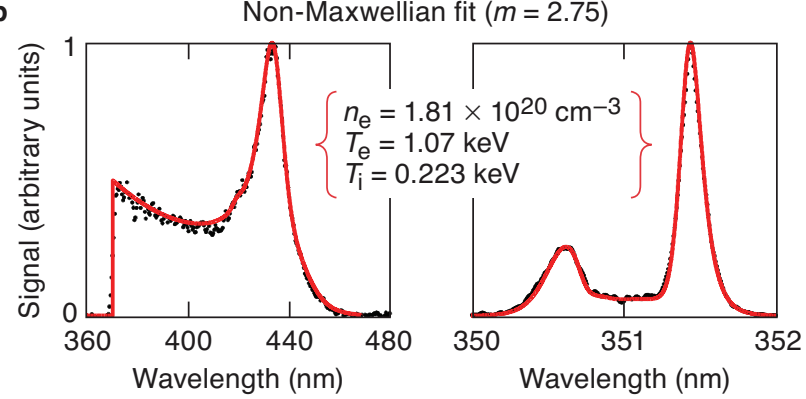

c

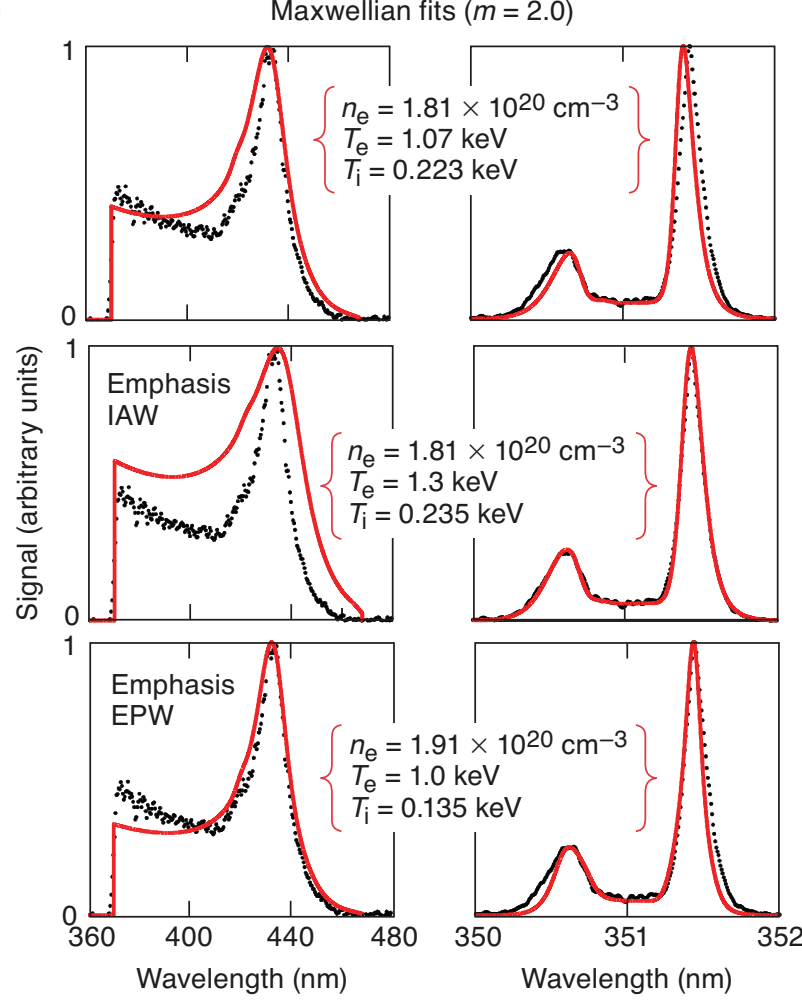

Figure 2: Thomson scattering results. (a) Timeresolved electron and ion features for the case of three heater beams (no pump). Lineouts were taken at 0.9 ns (bounded by the black dashed lines). (b) The best fit to both features indicated a super-Gaussian EDF of order $m=2.75$. (c) With the parameters extracted from the non-Maxwellian fit but a Maxwellian assumption, neither feature was reproduced. By adjusting density and temperature, an equivalent fit could be obtained for the ion feature, but electron features were discrepant. Conversely, minimizing the residuals of the electron features resulted in an inconsistent ion feature. In both cases, the plasma conditions inferred from the Maxwellian best fits resulted in $\varnothing(10 \%)$ errors in density and temperature. 
were therefore overlapping in wavelength. Figure 2(b) shows spectral measurements at $0.9 \mathrm{~ns}$ (points) along with the best fit (solid line). The super-Gaussian exponent in this case was determined to be $m=2.75$, which increases the frequency of IAW's by $\approx 9 \%$ over an equivalent Maxwellian plasma according to Eq. 4 .

To show clearly that a super-Gaussian EDF was required to fit these data, Maxwellian fits are shown in Fig. 2(c). Keeping the plasma conditions suggested by the non-Maxwellian fit, neither feature was reproduced accurately with the Maxwellian assumption (top row). Focusing only on reducing the ion feature discrepancy, an equally good fit could be obtained by increasing the electron temperature by $22 \%$; this came at the expense of the electron feature, which was then much broader than the data (middle row). Conversely, a better match to the electron feature (at the expense of the ion feature) was obtained by lowering the electron temperature and increasing the electron density (bottom row). The shape of the EPW feature was very sensitive to the EDF, however, and attempting to simultaneously match the peak location, width, and plateau region was not possible with the Maxwellian assumption. Note that both the ion feature degeneracy with respect to electron temperature and super-Gaussian exponent, as well as the ability for electron features to break that degeneracy, were previously predicted [21]. These errors in density and temperature that would result from measuring only the electron or the ion feature, along with a Maxwellian assumption, have significant ramifications for previous Thomson scattering experiments [21,22].

The results of the intensity scaling are shown in Extended Data Fig. 1 for the time indicated in Fig. 2. The superGaussian exponent measured by Thomson scattering (second to last row) increased with overlapped laser intensity in excellent agreement with Eq. 3. In each case, $m$ varied little over the 500-ps laser duration, suggesting the lasers maintain a quasi-steady-state EDF as long as they remain present. The last row contains the expected values that were computed using Eq. 3 along with the incident laser intensity averaged over the Thomson scattering volume $\left[I=N\left(5.1 \times 10^{14}\right) \mathrm{W} / \mathrm{cm}^{2}\right.$, where $N$ is the number of beams $]$ and the electron temperature measured in each case (which increased with laser intensity to the $\approx 0.2$ power). The agreement suggests that Eq. 3 can be used to compute EDF's when it is not possible to measure them directly.

Figure 3 shows the expected impact of the non-Maxwellian EDF on CBET for conditions similar to the 
a
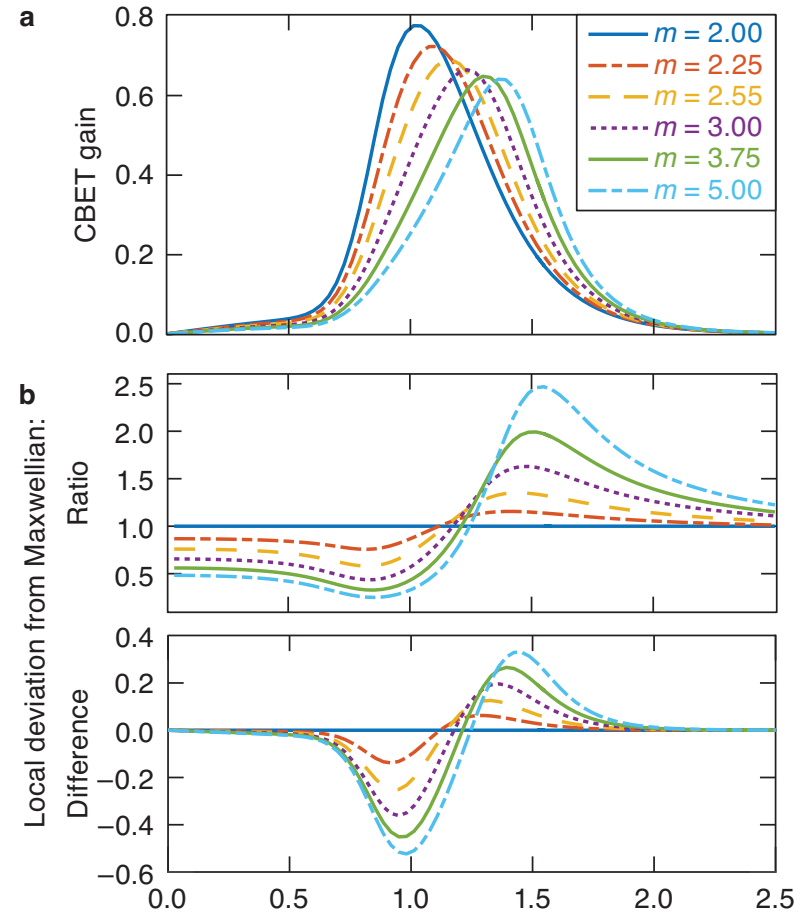

$\Delta \lambda(\AA ̊$ at $351 \mathrm{~nm})$

c

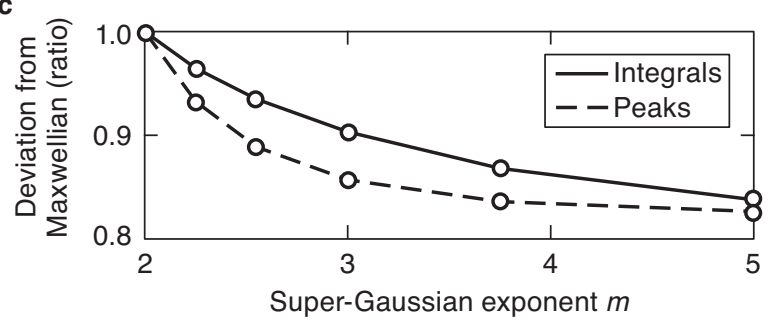

Figure 3: Effect of non-Maxwellian EDF on CBET gain.

(a) The idealized pump resonance is shown as a function of the non-Maxwellian super-Gaussian exponent. (b) The fractional deviation from the expected Maxwellian gain can be quite significant for fixed values of wavelength detuning, $\Delta \lambda$ (or probe wavelength shift relative to the pump beam, which is how CBET is typically controlled in indirect-drive ICF). The absolute difference in gain peaks in the vicinity of the Maxwellian resonance peak location. (c) The relative impact on the maximum gain, as well as the integral under each curve, is much smaller. 


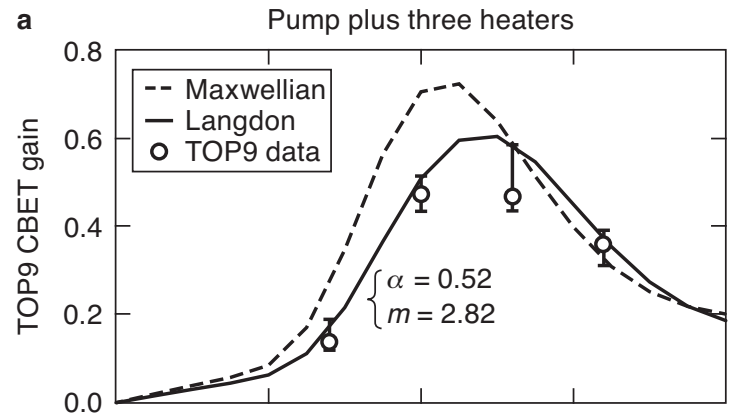

Figure 4: CBET results. (a) TOP9 data are shown for the case in which three heater beams coexisted temporally with the pump and wavelength-tunable beam. A calculation that accounts for the non-Maxwellian EDF

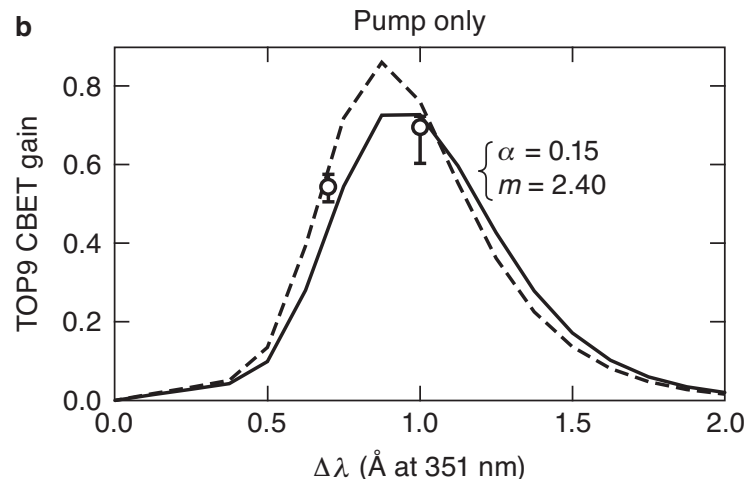
measured by Thomson scattering agrees with the data, but the Maxwellian calculation is discrepant. (See Methods for information concerning the origins of the error bars.) (b) Without the heater beams, the EDF was closer to Maxwellian and the data cannot easily distinguish between the two models.

TOP9 experiments (other than $m$, which is left as a free parameter). This calculation adapts the unpolarized linear kinetic CBET formulation [8], modified to use the numerically computed non-Maxwellian electron susceptibility as in the Thomson scattering analysis. The CBET gain-which relates the input and output probe intensities $G=\ln \left(I_{\text {out }} / I_{\text {in }}\right)$-is plotted against the wavelength shift of the probe beam in Fig. 3(a). The resonance peak shifts to higher frequency with increasing $m$ as expected from Eq. 4. Part (b) shows the local deviation from Maxwellian at every wavelength detuning position for each non-Maxwellian case as both a ratio (upper plot) and difference (lower plot); the fractional change is significant everywhere while the absolute difference in gain is maximized in the vicinity of the Maxwellian resonance peak. Since the maximum gain and the integral under each resonance curve do not change significantly [Fig. 3(c)], this effect is primarily a concern for situations where CBET is controlled via laser wavelength detuning (e.g., indirect-drive ICF).

Figure 4 shows data from the TOP9 experiments compared to simulations using the laser-plasma interaction code IFRIIT [23,24]. Part (a) contains results for the case in which the resonant pump plus three heater beams were present. The non-Maxwellian EDF measured by Thomson scattering must be accounted for in order to match the 


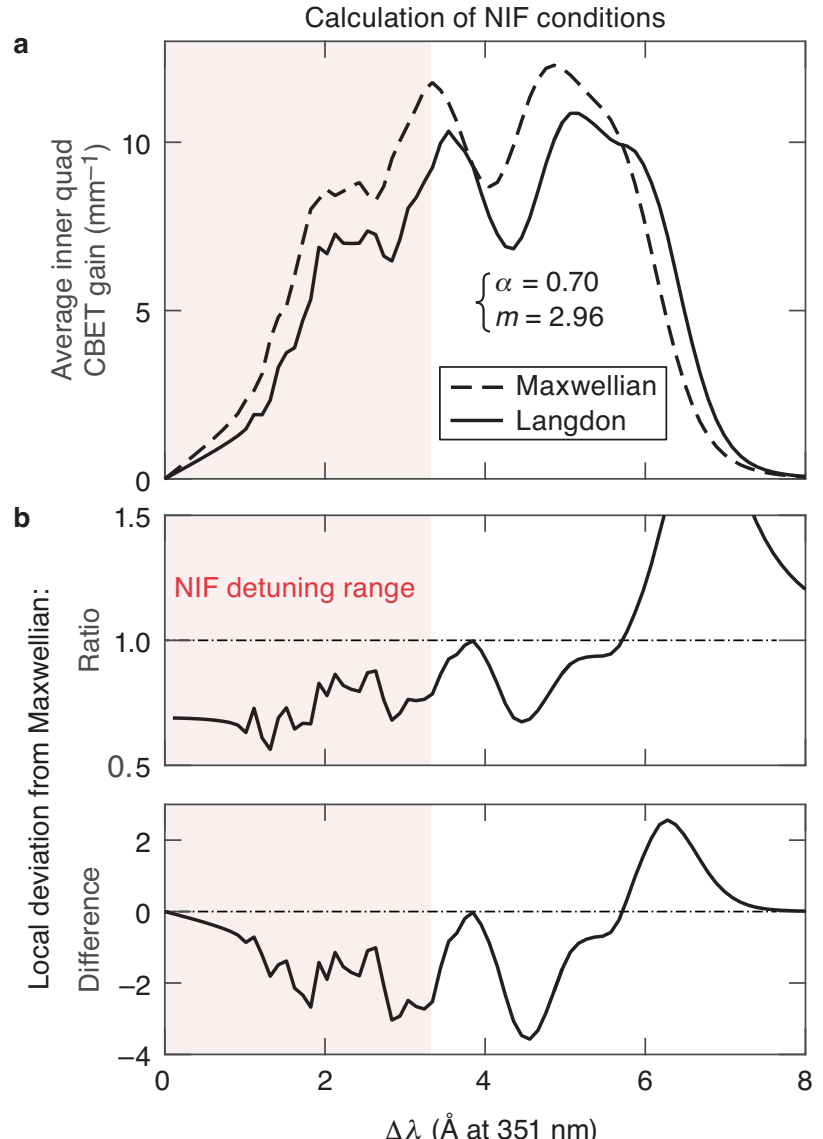

Figure 5: CBET calculation for NIF. (a) Gain per millimeter path length as a function of $\Delta \lambda$ (the wavelength difference between the inner and outer quads in this case) is shown for Maxwellian and non-Maxwellian distribution functions. Although additional structure results from summing over many resonances, the net effect of the nonMaxwellian EDF is similar to its impact on a single resonance. (b) The end result is that the Langdon effect suppresses CBET gain over NIF's entire wavelength tuning range.

measured CBET gain over the complete resonance. The expected plasma response assuming a Maxwellian electron distribution function (i.e., the linear kinetic model currently used in ICF calculations [5]) does not agree with the data. Note that the measured $m$ values in Fig. 4 were extracted from the Thomson scattering spectra on the actual CBET experiments (and $\alpha$ was inferred from $m$ using Eq. 3), and they are therefore slightly different from the results in Table 1, where no pump beam was present. See Methods for additional information regarding the CBET modeling.

Figure 4(b) shows results from two shots on which the heater beams were removed. In this case, the size of the Langdon effect driven by the pump alone is less significant ( $m=2.4 \pm 0.1$ was measured by Thomson scattering), and the data cannot easily distinguish between the Maxwellian and non-Maxwellian models.

Figure 5 shows a calculation of the expected impact of the Langdon effect on CBET in an indirect-drive fusion experiment at the NIF using plasma conditions $\left(T_{e}=2.8 \mathrm{keV}, T_{i}=0.8 \mathrm{keV}, Z=2\right)$ taken from the literature [8]. 
While both the lower atomic number of the helium fill gas and the higher hohlraum electron temperature reduce the Langdon parameter, the much higher overlapped intensity of the 96 beams (each at an assumed power of 2 TW) more than compensates, resulting in a predicted deviation from Maxwellian $(m=2.96)$ that is comparable to the TOP9 experiments. The plot in Fig. 5(a) is an estimate of the average gain experienced by an inner quad (beams that illuminate the hohlraum waist) given contributions from every outer quad (beams that illuminate the ends of the hohlraum), assuming a single value for the wavelength difference between the inner and outer beams. As is evident in Fig. 3(b), the higher IAW frequencies of the non-Maxwellian plasma suppress the gain on the rising edge of the resonance feature. This remains true when summing over many interactions, and, since most resonances are outside NIF's available wavelength detuning range ( 0 to $3.3 \AA$ ), the non-Maxwellian calculation for NIF is systematically lower [as highlighted by Fig. 5(b)]. Note that the assumption of fixed plasma conditions oversimplifies the conditions that govern CBET in an actual hohlraum, but this calculation was intended to capture the order-of-magnitude of the expected reduction. This level of CBET reduction would significantly impact implosion shape as well as reduce (and possibly eliminate) the need for a saturation clamp $[8,14]$. The non-Maxwellian CBET model could therefore greatly improve the predictive capability of integrated ICF modeling.

Of course, CBET in indirect-drive ICF is apt not to be the only process affected by non-Maxwellian EDF's in ICF plasmas and other laser-driven experiments. Rather, we expect that the Langdon effect is quite ubiquitous. A nonexhaustive list of other affected areas includes inverse bremsstrahlung absorption [1], heat transport [19,25-27], Thomson scattering analysis [21,22], other laser-plasma instability growth rates [3], the design of high-fluence plasma beam combiners [17], $\mathrm{x}$-ray spectroscopy analysis [2,27], magnetohydrodynamics [28], and other plasma diagnostics [29]. 


\section{Methods}

\section{TOP9}

TOP9 is an upgrade to the OMEGA EP laser that leverages the existing optical parametric amplification system in the short-pulse front end for spectrally broad amplification of a new, tunable, narrowband fiber front end [30]. After amplification and frequency tripling, a transport system image relays the beam to the P9 port of the OMEGA target chamber for joint operations with the OMEGA 60 -beam laser. The beamline delivers up to $0.5 \mathrm{TW}$ in pulses up to 1 ns in duration with a wavelength that is tunable from 350.2 to $353.4 \mathrm{~nm}$ [31].

\section{CBET experiments and analysis}

Energy conservation in CBET requires that there is a frequency difference between the interacting beams in the plasma rest frame. Energy is transferred from the higher frequency beam to the lower frequency beam, with the balance of the energy going into the ion acoustic wave. Although flow velocity can couple frequency-degenerate lasers (since the beams are Doppler-shifted in the moving frame of the plasma), controlled flows are difficult to produce and diagnose, making it hard to disentangle hydrodynamic uncertainty from CBET physics. In contrast, the ability to tune frequencies independently in the lab frame allows for energy transfer in stationary plasmas that are simpler to diagnose.

A 2-mm-outlet-diameter supersonic gas jet nozzle [32] released a mixture of $45 \%$ nitrogen and $55 \%$ hydrogen

gas (giving $Z_{\text {eff }}=6.11$ when fully ionized). For the first $500 \mathrm{ps,} \mathrm{nine} \mathrm{large-diameter} \mathrm{[850-} \mu \mathrm{m}$ full width at half maximum (FWHM)] plasma forming beams were overlapped at target chamber center in a quasi-symmetric fashion, each with $\approx 180 \mathrm{~J}$ of energy. Over the next $500 \mathrm{ps,} \mathrm{a} \mathrm{single} \mathrm{pump} \mathrm{beam} \mathrm{(with} \mathrm{an} \mathrm{angle} \mathrm{of} \mathrm{incidence} \mathrm{of} 21.41^{\circ}$ away from co-propagating with TOP9, an energy of $70 \mathrm{~J}$, and $127 \mathrm{GW}$ of power) was turned on along with TOP9 at a lower incident power of $10 \mathrm{GW}$. The wavelength of TOP9 was varied between shots in order to trace out the IAW 
resonance. To concentrate the interaction in the uniform region and increase the single pump CBET gain, smaller (163- $\mu$ m-diameter FWHM) phase plates were used for the TOP9 and pump beams. During this epoch, additional heater beams that nearly counter-propagate relative to TOP9 and overfill the interaction volume could be added to enhance the Langdon effect. These beams otherwise interacted minimally with the pump and TOP9 since TOP9 was frequency-tuned to resonantly drive small wave number ion acoustic waves (i.e., with co-propagating beams) but not the large wave number IAW's of counter-propagating beams. This was verified using a null shot with heater beams and TOP9 but no pump. All beams used polarization smoothing [33] with the exception of TOP9. For the final $500 \mathrm{ps}$, the pump was turned off but TOP9 remained on, providing a baseline measurement of plasma transmission. TOP9 terminated on a thin diffuser sheet inside the target chamber, the rescattered light from which was sampled in transmission by a CCD camera and a fiber-coupled streaked spectrometer (examples of the data from each diagnostic are shown in Fig. 1).

The incident TOP9 power and energy were measured using a streak camera and an absolutely calibrated calorimeter at a pickoff location prior to entering the target chamber. The streaked spectrometer in the TOP9 transmitted beam diagnostic was cross-calibrated by shooting through vacuum into the diagnostic. On CBET experiments, the transmitted power was impacted by energy transfer as well as inverse Bremsstrahlung absorption. To isolate the effect of CBET, the effect of absorption was determined by measuring transmission $T$ at a time after the pump turned off but while TOP9 remained on. The CBET gain was then found from $G=\ln \left(\frac{P_{\text {out }}}{P_{\text {in }}} \frac{1}{T}\right)$. Uncertainty in the transmission dominated the resulting uncertainty in CBET gain, and a range of plausible transmission values was used to determine the error bars in Fig. 4.

\section{Thomson scattering}

Thomson scattering spectra were calculated using the collisionless spectral density function [34],

$$
S(\mathbf{k}, \omega)=\frac{2 \pi}{k}\left(1-\frac{\chi_{e}}{\epsilon}\right)^{2} f_{e}\left(\frac{\omega}{k}\right)+\sum_{j} \frac{2 \pi}{k} \frac{p_{j} Z_{j}^{2}}{\bar{Z}}\left(\frac{\chi_{e}}{\epsilon}\right)^{2} f_{i}\left(\frac{\omega}{k}\right),
$$


where

$$
\chi_{e, i}(\mathbf{k}, \omega)=\int_{-\infty}^{\infty} d \mathbf{v} \frac{4 \pi q_{e, i}^{2} n_{e, i}}{M_{e, i} k^{2}} \frac{\mathbf{k} \cdot \partial f_{e, i} / \partial \mathbf{v}}{\omega-\mathbf{k} \cdot \mathbf{v}}
$$

and

$$
\epsilon=1+\chi_{e}+\sum_{j} \chi_{i, j} .
$$

The wave number and frequency of the probed fluctuations are related to the incident (subscript 0 ) and scattered (subscript s) light via $\mathbf{k}=\mathbf{k}_{0}-\mathbf{k}_{s}$ and $\omega=\omega_{0}-\omega_{s}$, respectively. The electron and ion distribution functions are $f_{e}$ (assumed to be in the form of Eq. 2) and $f_{i}$ (assumed to be Maxwellian), respectively. The summation in Eq. 5 is over all ion species $j$ where $p_{j}$ and $Z_{j}$ are the fraction and ionization state of each. The EDF factors in directly in the first term of Eq. 5 as well as indirectly via its slope, which enters $\chi_{e}$.

In practice, a synthetic spectrum is generated using Eq. 5 with plasma parameters as inputs, and it is then convolved with an instrument response function and compared to the data at a specific time. The inputs are iterated upon in order to minimize the residuals. In cases where multiple beams contributed to the Thomson scattering spectrum, their individual spectra were calculated using their respective scattering angles relative to the fixed Thomson scattering collection optics, and their relative intensities within the collection volume were used to produce a weighted summation. The electron feature was fit using only $n_{e}, T_{e}$, and super-Gaussian order $m$ as variables. Ion features were fit using $T_{e}, T_{i}$, and $m$ to reproduce peak separation and shape, as well as a relative drift velocity between the electrons and ions to reproduce the Stokes/anti-Stokes peak asymmetry.

The collection system used a Schwarzchild objective composed of two concentric spherical mirrors coupled to a Pfund objective to transport scattered light from a $\approx 100 \mu \mathrm{m}$ volume at target chamber center to a pair of streaked spectrometers with diffraction-limited performance across all measured wavelengths. Ion acoustic features were measured with 0.02-nm spectral resolution and 200-ps temporal resolution using a 1-m Czerny-Turner spectrometer outfitted with a 2160-lines/mm grating and coupled to a Rochester optical streak system (ROSS). Electron plasma wave features were measured with 0.5 -nm spectral resolution and 50-ps temporal resolution using a $0.3-\mathrm{m}$ spectrometer (150lines $/ \mathrm{mm}$ ) coupled to a second ROSS [35]. The ion features were removed from the electron feature measurement 
using a spectral notch filter to avoid saturating the streak camera.

\section{CBET modeling}

The CBET interaction was modeled using the IFRIIT laser-plasma interaction code $[23,24]$. IFRIIT solves the firstorder Debye expansion of the Helmholtz equation for the electric field in 3-D geometries. Inverse ray tracing of geometrical optics rays is used to reconstruct the phase and amplitude of the various wavefields in the plasma, including the effects of refraction, inverse Bremsstrahlung absorption, and refraction-induced field swelling. CBET is taken into account by adding a perturbation term $\delta \epsilon$ to the permittivity seen by each wavefield. Here, $\delta \epsilon$ is computed using the unpolarized linear kinetic formulation [8]. The unpolarized model assumption was verified against more detailed calculations including the detailed spot separation induced by distributed polarization rotators. The electron susceptibility $\chi_{e}$ was computed from the derivative of the plasma dispersion function, using the same tabulation for the non-Maxwellian EDF as in the Thomson scattering calculations. The super-Gaussian order $m$ was computed inline within each mesh cell from the Langdon parameter $\alpha$, using Eq. 3. The surface of the 3-D interaction volume was adaptively refined in order to ensure energy conservation of the calculation, and pump depletion was accounted for by iteration of the wavefield phase calculation.

The complex electric field of each beam was described with a plane wavefront and prescribed 2-D intensity distributions. Given the sensitivity of the CBET interaction to laser intensity, particular care was given to characterizing the laser spot profiles. All beams-the co-propagating seed and pump as well as the counter-propagating Langdon heater beams-were included in the simulations using far-field spatial profiles measured by equivalent target plane images. The plasma was described using a spherical density profile of super-Gaussian shape, whose radius and order were extracted from separate experiments in which the Thomson scattering data was spatially resolved along the axis of the probe beam in lieu of temporal resolution. The radial density profile, which was nearly homogenous, impacted the results negligibly. Electron and ion temperatures were assumed homogeneous and corresponded to the measured values from Thomson scattering fits at target chamber center. These assumptions were justified given the small size of 
the interaction volume compared to the extent of the homogenous plasma region.

\section{Data availability}

The data represented in Fig. 2(b-c) are available as source data in Supplementary Data 2. All other data that support the plots within this paper and other findings of this study are available from the corresponding author upon reasonable request.

\section{Code availability}

The computer programs that support the findings of this study are available from the corresponding author upon reasonable request.

\section{Extended Data}

[1] Langdon, A. B. Nonlinear inverse bremsstrahlung and heated-electron distributions. Physical Review Letters 44, 575-579 (1980)

[2] Matte, J. P. et al. Non-maxwellian electron distributions and continuum X-ray emission in inverse bremsstrahlung heated plasmas. Plasma Physics and Controlled Fusion 30, 1665-1689 (1988).

[3] Afeyan, B. B., Chou, A. E., Matte, J. P., Town, R. P. J. \& Kruer, W. J. Kinetic theory of electron-plasma and ion-acoustic waves in nonuniformly heated laser plasmas. Physical Review Letters 80, 2322-2325 (1998).

[4] Betti, R. \& Hurricane, O. A. Inertial-confinement fusion with lasers. Nature Physics 12, 435-448 (2016). 


\begin{tabular}{|c|c|c|c|c|}
\hline & 1 beam & 2 beams & 3 beams & 4 beams \\
\hline$I\left(10^{14} \mathrm{~W} / \mathrm{cm}^{2}\right)$ & $5.1 \pm 0.2$ & $10.2 \pm 0.2$ & $15.3 \pm 0.3$ & $20.4 \pm 0.3$ \\
\hline$T_{e}(\mathrm{keV})$ & $0.84 \pm 0.03$ & $0.98 \pm 0.03$ & $1.07 \pm 0.03$ & $1.10 \pm 0.03$ \\
\hline$\alpha$ & 0.17 & 0.30 & 0.41 & 0.53 \\
\hline$m$ & $2.40 \pm 0.05$ & $2.65 \pm 0.05$ & $2.75 \pm 0.05$ & $2.85 \pm 0.05$ \\
\hline$m_{\text {calc. }}$ & $2.43 \pm 0.01$ & $2.60 \pm 0.01$ & $2.72 \pm 0.02$ & $2.83 \pm 0.02$ \\
\hline
\end{tabular}

Figure 1: Langdon effect intensity scaling plasma characterization results. Different experiments were conducted in which the number of beams present after an initial plasma-forming period was varied from 1 to 4 -each having nominally identical power and spatial smoothing. Overlapped intensity therefore scaled linearly with the number of beams. This resulted in a more than threefold increase in the Langdon parameter $(\alpha)$ despite the moderating influence of the higher electron temperature. In each case, the super-Gaussian exponent, $m$, of the electron distribution function measured by Thomson scattering was found to be in excellent agreement with the value expected from Eq. 3 .

[5] Michel, P. et al. Tuning the implosion symmetry of icf targets via controlled crossed-beam energy transfer. Physical Review Letters 102, 025004 (2009).

[6] Glenzer, S. H. et al. Symmetric inertial confinement fusion implosions at ultra-high laser energies. Science 327, 1228-1231 (2010).

[7] Moody, J. D. et al. Multistep redirection by cross-beam power transfer of ultrahigh-power lasers in a plasma. Nature Physics 8, 344-349 (2012).

[8] Michel, P. et al. Stochastic ion heating from many overlapping laser beams in fusion plasmas. Physical Review Letters 109, 195004 (2012).

[9] Clark, D. S. et al. Three-dimensional simulations of low foot and high foot implosion experiments on the national ignition facility. Physics of Plasmas 23, 056302 (2016).

[10] Strozzi, D. J. et al. Interplay of laser-plasma interactions and inertial fusion hydrodynamics. Physical Review Letters 118, 025002 (2017). 
[11] Berzak Hopkins, L. F. et al. First high-convergence cryogenic implosion in a near-vacuum hohlraum. Physical Review Letters 114, 175001 (2015).

[12] Hinkel, D. E. et al. Development of improved radiation drive environment for high foot implosions at the national ignition facility. Physical Review Letters 117, 225002 (2016).

[13] Baker, K. L. et al. High-performance indirect-drive cryogenic implosions at high adiabat on the national ignition facility. Physical Review Letters 121, 135001 (2018).

[14] Kritcher, A. L. et al. Energy transfer between lasers in low-gas-fill-density hohlraums. Physical Review E 98, 053206 (2018).

[15] Kirkwood, R. K. et al. Observation of energy transfer between frequency-mismatched laser beams in a largescale plasma. Physical Review Letters 76, 2065-2068 (1996).

[16] Turnbull, D. et al. Refractive index seen by a probe beam interacting with a laser-plasma system. Physical Review Letters 118, 015001 (2017).

[17] Kirkwood, R. K. et al. Plasma-based beam combiner for very high fluence and energy. Nature Physics 14, 80-84 (2018).

[18] Dum, C. T. Anomalous heating by ion sound turbulence. Physics of Fluids 21, 945-955 (1978).

[19] Dum, C. T. Anomalous electron transport equations for ion sound and related turbulent spectra. Physics of Fluids 21, 956-969 (1978).

[20] Liu, J. M., De Groot, J. S., Matte, J. P., Johnston, T. W. \& Drake, R. P. Measurements of inverse bremsstrahlung absorption and non-maxwellian electron velocity distributions. Physical Review Letters 72, 2717-2720 (1994).

[21] Zheng, J., Yu, C. X. \& Zheng, Z. J. Effects of non-maxwellian (super-gaussian) electron velocity distribution on the spectrum of thomson scattering. Physics of Plasmas 4, 2736-2740 (1997). 
[22] Milder, A. L., Ivancic, S. T., Palastro, J. P. \& Froula, D. H. Impact of non-maxwellian electron velocity distribution functions on inferred plasma parameters in collective thomson scattering. Physics of Plasmas 26, 022711 (2019).

[23] Colaitis, A., Palastro, J. P., Follett, R. K., Igumenschev, I. V. \& Goncharov, V. Real and complex valued geometrical optics inverse ray-tracing for inline field calculations. Physics of Plasmas 26, 032301 (2019).

[24] Colaitis, A., Follett, R. K., Palastro, J. P., Igumenshchev, I. \& Goncharov, V. N. Adaptive inverse ray-tracing for accurate and efficient modeling of cross beam energy transfer in hydrodynamics simulations. Physics of Plasmas 26, 072706 (2019).

[25] Epperlein, E. M. \& Short, R. W. Nonlocal electron transport in the presence of high-intensity laser irradiation. Physical Review E 50, 1697-1699 (1994).

[26] Batishchev, O. V. et al. Heat transport and electron distribution function in laser produced plasmas with hot spots. Physics of Plasmas 9, 2302-2310 (2002).

[27] Barrios, M. A. et al. Developing an experimental basis for understanding transport in nif hohlraum plasmas. Physical Review Letters 121, 095002 (2018).

[28] Bissell, J. J., Ridgers, C. P. \& Kingham, R. J. Super-gaussian transport theory and the field-generating thermal instability in laser-plasmas. New Journal of Physics 15, 025017 (2013).

[29] Seka, W. et al. Nonuniformly driven two-plasmon-decay instability in direct-drive implosions. Physical Review Letters 112, 145001 (2014).

[30] Dorrer, C. et al. Spectrally tunable, temporally shaped parametric front end to seed high-energy nd:glass laser systems. Optics Express 25, 26802-26814 (2017).

[31] Kruschwitz, B. E. et al. Tunable uv upgrade on omega ep. SPIE High Power Lasers for Fusion Research V Proceedings 10898, 1089804 (2019). 
[32] Semushin, S. \& Malka, V. High density gas jet nozzle design for laser target production. Review of Scientific Instruments 72, 2961-2965 (2001).

[33] Boehly, T. R. et al. Reduction of laser imprinting using polarization smoothing on a solid-state fusion laser. Journal of Applied Physics 85, 3444-3447 (1999).

[34] Froula, D. H., Glenzer, S. H., Luhmann, N. C. \& Sheffield, J. Plasma Scattering of Electromagnetic Radiation (Academic Press, Boston, 2011), 2nd edn.

[35] Katz, J. et al. A reflective optical transport system for ultraviolet thomson scattering from electron plasma waves on omega. Review of Scientific Instruments 83, $10 \mathrm{E} 349$ (2012).

Acknowledgments This material is based upon work supported by the Department of Energy National Nuclear Security Administration under Award Number DE-NA0003856, the University of Rochester, and the New York State Energy Research and Development Authority. This report was prepared as an account of work sponsored by an agency of the U.S. Government. Neither the U.S. Government nor any agency thereof, nor any of their employees, makes any warranty, express or implied, or assumes any legal liability or responsibility for the accuracy, completeness, or usefulness of any information, apparatus, product, or process disclosed, or represents that its use would not infringe privately owned rights. Reference herein to any specific commercial product, process, or service by trade name, trademark, manufacturer, or otherwise does not necessarily constitute or imply its endorsement, recommendation, or favoring by the U.S. Government or any agency thereof. The views and opinions of authors expressed herein do not necessarily state or reflect those of the U.S. Government or any agency thereof.

Author Contributions DT - P.I. for the experimental campaign; AC - theory and modeling P.I.; AMH - experimental support and plasma characterization; ALM - non-Maxwellian electron susceptibility calculations; JPP - theory support; JK - instrument specialist; CD and BEK - laser specialists; DJS - motivational discussions and supporting simulations; DHF - experimental plasma physics group leader

Competing Interests The authors declare no competing interests.

Correspondence Correspondence and requests for materials should be addressed to D.T. (email: turnbull@ lle.rochester.edu). 